\title{
Bibliatbèque
}

\section{D." Dauquetion}

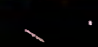


काषicis a cramara a coseres

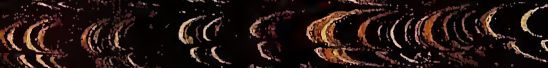

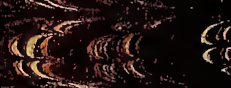

4

(c)

(ic) ac resis (C) Tilas (c) a. \& $d \pi^{\circ}$

efrac

बराC o canc. as

cric $=$ re icic

\& C CL

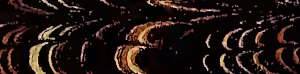<smiles>C1=C[Ge][GeH]=C1</smiles>

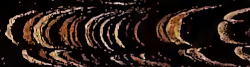
Corratich

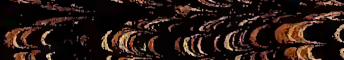

$6\left(\int\right)(0)$

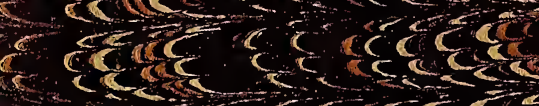

os k

\section{esecte \\ siscec}

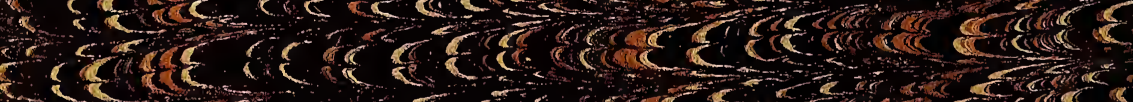

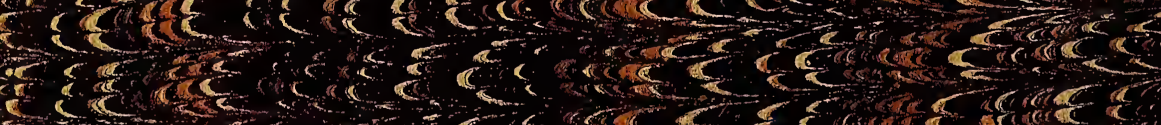

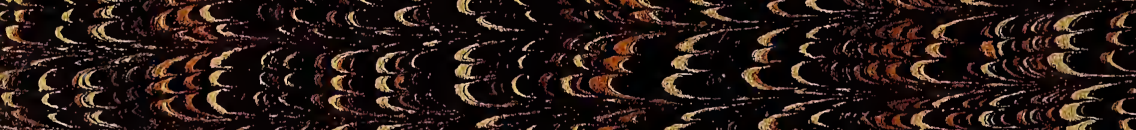

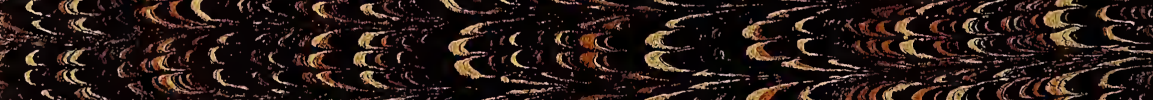

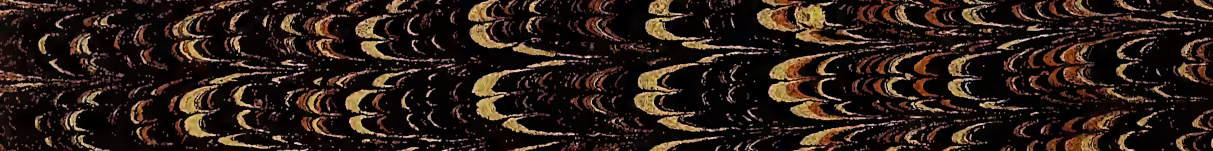

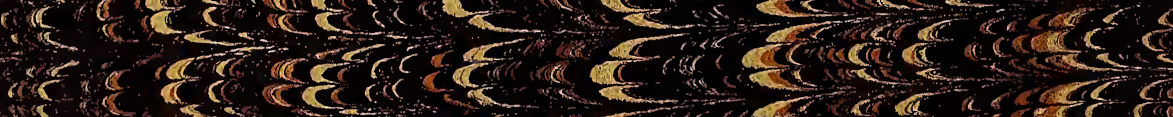

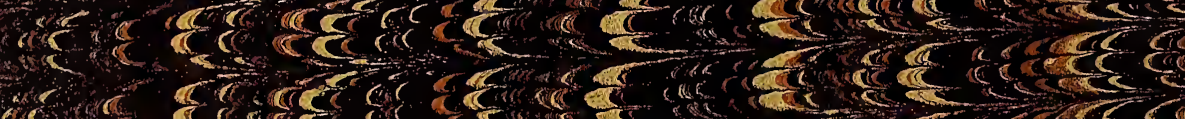

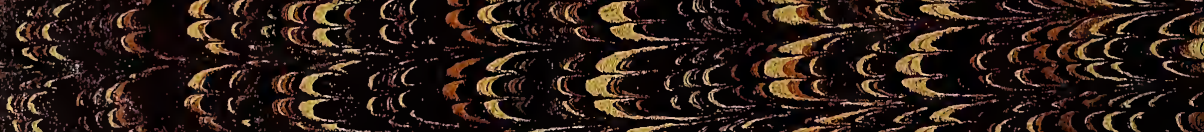

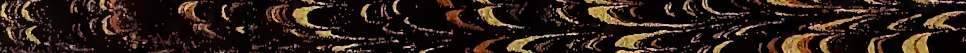



An $2 b$ 






\section{MEMOIRE}

SUR QUELQUES COQUILLES FOSSILES NOUVELLES,

DÉCOUVERTES DANS IA RÉGION AQUTTANIQUE DU BASSIN SOUS-PYRÉNÉEN;

Par M. MOULET.

Depurs quelques années, le bassin sous-pyrénéen est devenu un vaste champ de recherches paléontologiques; mais jusqu'ì ce jour, les savants se sont beaucoup pluś préoccupés des animaux vertébrés qui y ont été découverts, que des coquilles fossiles que l'on y rencontre, et qui forment, il faut le reconnaître, au point de vue géologique, une des sections les plus intéressantes de la Faune de celte circonscription naturelle. L'Académie des Sciences de Toulouse l'avait ainsi compris, en proposant pour sujet du prix de cette année (1846), une question ayant pour but d'attirer plus particulièrement l'attention des naturalistes vers l'étude des mollusques fossiles de ce vaste espace.

Avant que les luttes du concours, prêt à s'ouvrir, soient engagées, je crois utile, dans l'intérêt des travaux scientifiques qui seront entrepris à ce sujet, de faire connaître quelques faits intéressants, qui pourraient être omis ou négligés faute de renseignements précis. Je bornerai néanmoins mes communications actuelles à décrire quelques coquilles des genres Mélanic (Mclania) et Mulette (Unio), provenant toutes de celte portion du bassin sous-pyrénéen (1) qui est enclavée dans les

(1) Tout l'espace que nous avons, le premier, compris sous la dénomination générale de Bassin sous-pyrénéen, est occupé par un terrain d'eau 3. ${ }^{\mathrm{S}}$ S. - TOME II. 
limites de l'antique Aquitaine sous la domination romaine. Cet espace ne comprend qu'une petite partie du département de la Haute-Garonne, et la presque totalité de celui du Gers. II est géognostiquement constitué par des roches tendres : argiles, marnes, sables et grès-molasses, horizontalement disposés en lits ou strates ayant peu d'épaisseur, passant de l'un à l'autre sans aucune alternance régulière et suivic. Les sables et les grìs se montrent assez fréquemment en amas plus ou moins puissants. Ces diverses couches et ces dépôts, mais principalement les arénacés, renferment des ossements caractérisant de nombreux animaux vertébrés dont les races sont éteintes.

Dans la partie inférieure du Gers, et en descendant dans l'ancien Agenais, se montrent, mais dépendants de la grande formation marno-sableuse que nous venons d'indiquer, des calcaires d'eau douce, le plus souvent stratifiés, à disposition horizontale.

Les deux termes de celte grande formation, les roches marnosablezses et les calcaires, contiennent des coquilles. fossiles. Le côté neuf de ce sujet réside dans la distinction de celles qui sont particulières à chacun des deux terrains. Je commence par celles qui me semblent propres principalement aux sables et aux grès-molasses, mais que l'on trouve aussi, quoique fort rarement, dans les couches d'argile et de marne. Ces coquilles manquent dans les calcaires proprement dits, et ceux-ci présentent une population de mollusques distincte.

Il m'a semblé qu'un intérêt plus grand devait s'attacher à l'étude des mollusques qui ont accompagné dans leur dispersion et leur délaissement, les débris de nos animaux fossiles vertébrés, ce qui m'a déterminé à commencer par ceux-ci. Jé signalerai comme caractéristiques du terrain marno-sableux,

douce qui rentre dans l'étage mojen (Miocène) des terrains tertiaires. Le centre et la partie supérieure sont uniquement constitués par des dépóts marno-sableux; ce n'est qu'à la marge sur quelques points que se montrent des calcaires, en formant une sorte de ccinture, fréquemment interrompue. 
dans la circonscription aquitanique, une roquille du genre Mélanie et plusieurs espèces du genre Mulelte.

Les Mélanies actuelles vivent dans les eaux douces, plus particulièrement dans les eaux courantes. On n'en a point découvert en France; la plupart sont même étrangères à l'Europe. On en trouve fréquemment à l'état fossile. Ce genre a été ainsi caractérisé, daaprès le test :

\section{Genre MEL $A N I A$. Lamk. MÉlaANIE.}

Coquille turriculée. Ouverture entière, ovale ou oblongue, évasée à la base. Columelle lisse, arquée en dedans. Un opercule cornë.

1. Mélanie D’Aquitane. Melania aquitanica. Noul.

$$
\text { Pl. } 1 \text {, fig. 1, } 2 .
$$

M. Tesiâ elongato-pyramidale, acutâ, longitudinaliter. cosıatá; anfraclibus subplanis, suprà obliquè spiratis; sulcis subimbricatis; aperturấ ovatâ.

Cette coquille est allongée, turriculée, pyramidale, terminéc en pointe aiguë. La spire est formée de 10-11 tours, larges, aplatis, ou à peine convexes, pourvus de côtes (10-12) légèrement obliques, régulièrement espacées, se continuant jusqu’à la carène; ces tours sont traversés par des sillons comme imbriqués ( $3-4-5)$, le plus souvent inégaux en largeur, finissant sur les côtes en tubercules d'abord aigus, puis obtus, arrondis. Le dernier tour est sillonné, mais les côtes disparaissent vers le milieu. Les tours sont étagés par une rampe oblique, large, que l'on voit à leur partic supérieure; elle est occupée par des stries plus fines que celles des tours, et ne présente point de cótes. L'ouverture est ovale, évasée à la base.

La coquille de celte espèce était épaisse et noire (1), comme le démontrent les rares exemplaires que l'on rencontre ayant con-

(a) Lamark a tiré le nom du genre Mélanie de la couleur habituellement noire des coquilles vivantes qui le constituent. 
servé leur test. L’ouverture manque constamment dans tous ceux que nous avons vus dans cet état. Nous avons rétabli cette portion si importante de la coquille d'après les empreintes que nous ont fournies plusicurs moules extérieurs. Ces moules sont excessivement répandus dans le département du Gers, et dans une partie de celui de la Haute-Garonne; ils occupent, en creux, le centre de concrétions pierreuses fort dures, que l'on cmploie fréquemment au pavage des cours, à l'empierrement des routes et à la bâtisse grossière. Ce sont des sortes de géodes, composées de couches argilo-calcaires superposées, auxquelles le test de la coquille avait servi primitivement de noyau. Celui-ci ayant fini par disparaître, il ne reste plus que le moule exact de la forme extérieure de la coquille, que l'on rétablit parfaitement en en retirant des empreintes. Au reste, le phénomène que nous décrivons se produit de nos jours, sous nos yeux, et jusque dans les mêmes localités d'où proviennent les moules de nos fossiles : nous avons rapporté du Gers bon nombre de coquilles fluviatiles actuelles, recouvertes d'un dépôt souvent épais de substance calcaire.

La taille de la Mćlanie d'Aquitaine était variable; les plus grands excmplaires atteignent 6-7 centimètres de hautcur ; souvent, les deux, trois et quatre derniers tours manquent, et alors le sommet est plus ou moins obtus et comme tuberculé. Cette coquille est aussi très-variable sous le rapport de la disposition de ses côtes et des tubercules qui les surmỏntent, et qui, par l'effet de l'usure, finissent toujours par être plus ou moins effacés à la longue, ce que l'on observe surtout dans les premiers tours.

Nous avons déjà dit que cette coquille était très-répandue ; mais c'est plus particulièrement dans les lits ou dépôts arénacés qu'on la trouve, quoiqu'elle se présente aussi dans les couches argileuses et marneuses : à Montferran (Gers), elle gît dans une argile à peine calcarifère où le test s'est conservé; il en est de même à Goutz, près de Fleurance.

Un fait important à noter, c'est que notre Mélanic occupe des points differents dans les profondeurs du terrain miocène; 
nous l'avons rencontrée sur le sommet des collines les plus élevées, sur la crète de coteaux secondaires, sur lcurs flancs à diverses hauteurs, et jusqu'au niveau des cours d'eau qui sillonnent la contrée où elle abonde (1). Au reste, elle accompagne le plus souvent les Mulettes qui vont nous occuper, et des débris de divers mammifères : à Castillon (Gers), nous l'avons observée abondante, dans une molasse à gros grains avec des os de Mastodon angustidens et des moules de Mulettes plissées; au Pin (Haute-Garonne), elle gisait dans les sables avec des restes de Rhinocéros et du Palcotherium hyppoïdes.

Voici les localités précises dans lesquelles la présence de la Mélanic d'Aquitaine a été constatée :

1. Entre la vallée de la Garonne et celle de la Save, à Mondavezan, à Benque (Haute-Garonne); à Puylausic, à Montpezat (Gers); au Pin (Haute-Garonne); à Gensac, à Laymont, à Périgué, à l'Isle-en-Jourdain, à Pujaudran (Gers).

2. ${ }^{\circ}$ Entre la vallée de la Save et celle de la Gimone, à Lahas, à Nizas, à Castillon, à Montferran, à Cologne (Gers) avec les Mulettes.

3. Entre la Gimone et le Gers, à Castelnau-Barbarens, à Pessan, à Seissan, à Goutz (Gers).

4. ${ }^{\circ}$ Entre le Gers et la Baïse, à Castera-Verduzan (Gers).

Cette coquille est donc très-répanduc dans une grande étendue de l'ancienne Aquitaine, et mérite la dénomination spécifique que nous lui avons donnée.

Les premiers géologistes, MM. Brongniard et de Férussac, qui caractérisèrent si heureusement les terrains d'eau douce supérieurs à la craie, à l'aide des coquilles fossiles qu'ils recélaient, exprimèrent leur surprise de ne pas y avoir rencontré des genres bivalves.'

(1) Les eaux de la Save roulent des géodes Mélanifères; on en trouve fréquemment dans les sables sur les bords de celte pelite rivière. 
Postéricurement aux travaux de ces savants, M. Marcel de Serres crut reconnaître quelques Mulettes dans les couches tertiaires du Midi de la France, sans être parvenu, toutefois, à les déterminer rigoureusement (1). M. Bouillet a décrit une Mulette commune dans l'argile verdâtre du fond d'un ancien lac, au Nord de la montagne de Georgovia en Auvergne (2); M. de Blainville a cité une espèce du genre Unio venant du Gers, que lui avait communiquéc M. Lartet, et que le savant malacologiste considéra comme très-rapprochée, sinon identique à l'Unio margaritifera. Lumк., sans faire attention qu'elle était plisséc ou côtelée à sa surface (3). Enfin, M. l'abbé Dupuy a signalé une coquille du même genre, découverte par notre ami commun M. L. Lacaze, près de Cologne (Gers) (4). Tels sont, je crois, les faits peu nombreux touchant la présence des coquilles du genre Unio dans les formations tertiaires de la France et en particulier dans le bassin sous-pyrénéen. C'est donc une bonne fortune que d'avoir à annoncer que le même terrain qui nous a fourni la Mélanie d'Aquitaine, renferme neuf espèces de Mulettes, nouvelles pour la science.

Les Mulettes vivantes sont très-répanducs dans les caux fluviales; elles se tiennent dans la vase, ayant leurs sommets dirigés en bas ; et ainsi groupées, souvent en très-grand nombre, clles résistent à la rapidité des courants. Mortes, leurs coquilles sont entraînées et dispersées sur les bords, ou enfouies dans les terrains alluviens, jusqu'à de très-grandes distances de leur point de départ. On en trouve aussi dans les lacs alimentés par des cours d'eau puissants, ainsi que cela a licu dans l'Amérique du Nord.

Nos espèces fossiles appartiennent aux vraies Mulcttes, à celles que caractérise une charnière compliquée de dents longi-

(1) Géognosie des terr. tert. 1829 , in-8. ${ }^{\circ}$, pag. 143 .

(2) Cat. des coq. viv. et foss. de l'Auvergne. 1836. in-8. ${ }^{\circ}$ pag. 149.

(3) Comptes-rendus des séances de l'Acad. des Scienc. (1837), t. 5, p. 426.

J'ai fait la reclification spécifique d'après les renseignements que m’a fournis M. Larlet (in litt.).

(4) Essai sur les coq. viv. et foss. du Gers. 18,3 , in-8. ${ }^{\circ}$ pag. 102. 
tudinales et cardinales. Leur facies général permet de les séparer en deux groupes tranchés: l'un comprend les espèces analogues aux Mulettes d'Europe, à celles-là même qui vivent dans le bassin sous-pyrénéen (1); l'autre est remarquable par les plis ou côtes que présentent les coquilles. Celles-ci trouvent leur place, dans la série naturelle, parmi les Mulettes plissées que l'on rencontre dans les grandes rivières et dans les lacs de l'Amérique septentrionale.

Tous ceux qui se sont occupés de l'étude des Mulettes vivantes, conviennent de l'arbitraire qui règne dans la délimitation des espèces; la forme et la taille des coquilles varient infiniment, suivant l'âge et aussi suivant les conditions d'existence dans lesquelles l'animal a vécu. Toutefois, c'est là un léger inconvénient, pourvu que les différences constantes et facilement saisissables, qui les distinguent, soient exactement signalées. D'après ces vues, j’ai nommé et décrit, comme espèces, toutes les coquilles fossiles qui mont offert des caractìres nettement définis, quels que fussent leurs rapports prochains avec les espèces voisines. Je pense que nous aurons à établir postérieurement quelques espèces de plus; divers fragments de ma collection, mais trop incomplets pour fournir des diagnoses suffisantes, ne me laissent aucun doute à cet égard.

Ce que j’ai dit précédemment du gisement de la Mélanic d'Aquitaine est de tout point applicable aux Mulettes, puisque ces coquilles se trouvent habituellement ensemble, enfouies dans les mêmes couches. Je dois pourtant noter qu'on n'a point encore rencontré de Mulettes ayant conservé leur test; leur présence n'a été jusqu'ici constatée que par les moules qu'ont laissés leurs coquilles détruites, le plus souvent dans des géodes semblables à celles qui ont conservé l'empreinte de la Mélanie, très-rarement dans des grès à petits grains et solides (à Cologne, Gers).

Les géodes qui renferment les empreintes de nos Mulettes ont été le plus souvent observées près de la surface du sol dans les

(1) Noulet, Précis analyı. de l'llist. des Mollusques du Bass, sons-pyrénéen. เ834, in-8. - Dupuy, 1. c. 
couches ou dépôts sableux sur la crête des collines où les travaux de l'homme les mettent facilement à découvert : au Planté, au Pin, à Laymont, à Lahas, à Nizas, à Pessan, à Simorre, elc. Mais on les trouve en place dans des couches encore intactes, sur le flanc de ces mêmes collines; j'en ai trouvé moi-même au Pin, et en allant de Benque à Saint-Frajou ; enfin , on les rencontre dans des lieux plus bas, au pied des coteaux : à Cologne, à Monferran, à Castillon, etc.

Les espèces que nous allons décrire ne se montrent pas isolées, on les rencontre toujours plusieurs ensemble, et dans le même gisement, les Mulettes plissées accompagnent celles qui ne présentent pas de plis à leur surface. Enfin, ce sont des valves dépareillées que nous trouvons presque toujours à l'état d'empreintes, et ce n'est que par exception qu'on les rencontre réunies deux à deux pour compléter le moule de la coquille. Souvent on n'a à faire qu'à des fragments de valve.

Tels sont les faits principaux que j'ai cru devoir reiater avant de chercher à caractériser spécifiquement les Mulettes fossiles que des recherches longtemps continuées m'ont fait découvrir dans notre terrain miocène, aidé de la précieuse collaboration de plusieurs de mes amis, et principalement de M. L. Lacaze et des Docteurs Seré, Fazeuilhe et Barrère : je les pric d'agréer ici toute ma reconnaissance.

Gexne UNIO. Retz. MULETTE.

Coquille bivalve; charnière ì deux dents sur chaque valve; l'une cardinale irrégulière simple ou divisée en deux, l'autre allongée, se prolongeant sous le corcelet; ligament cxtérieur.

\section{A. Plicati.}

1. Mulette ex eventall. Unio flabelliferus. Noul.

$$
\text { PI. } 2 \text {, fig. } 1 .
$$

U. Teslâ ovato-oblongâ, crassâ, plicatâ; plicis latis, pluribus summo in uno confertis, à natibus ad marginem posteriorem rectò decurrentibus, plicis alteris numerosis, recurvis, ad marginem superiorem et postcriorem decur- 
rentibus; natibus prominentibus; margine inferiore subsinuato, margine superiore leviter arcuato; dente cardinali crasso, conico, obtuso, sulcato.

$$
\begin{aligned}
& \text { Alt. } 0^{\mathrm{m}}, 05 . \\
& \text { Lat. } 0,1 \text {. }
\end{aligned}
$$

Coquille ovale-allongéc, assez épaisse et régulièrement convexe, relevée à sa partic postérieure de plis coniques disposés en éventail; les uns très-marqués, naissent en commun au-dessous des sommets et vont en s'irradiant directement vers les bords postérieur et inférieur; les autres, un peu courbes, sont obliquement dirigés d'avant en arrière jusqu'aux bords supérieur et postérieur, occupant ainsi la place du corcelet (1). Le sommet est peu proéminent et placé très-en avant. Le bord supérieur est peu arqué; l'inféricur est légèrement sinué, les stries d'accroissement sont espacées. Des fragments de moule me font penser que cette coquille atteignait quelquefois des dimensions plus fortes que celles que je lui ai attribuées d'après le moule figuré.

Localités: Mondavezan, le Pin, le Planté, Laymont, Lahas, Nizas , etc.

2. Mulette a plis courts. Unio breviplicatus. Noul. Pl. 3, fig. 1.

U. Testâ ovatâ, crassâ, ventricosâ, plicatâ; plicis 4 summo in uno confertis, à natibus ad marginem inferiorem et posteriorem decurrentibus; plicis alteris numerosis, recurvis, ad marginem superiorem et posteriorem decurrentibus; natibus prominentibus; margine inferiore sinuato, subcrenulato, margine superiore arcuato; dente cardinali crasso, conico-triangulari, obtuso, sulcato.

$$
\begin{array}{ll}
\text { Alt. } 0^{\mathrm{m}}, & 038 . \\
\text { Lat. } 0, & 07 .
\end{array}
$$

(1) On trouve cette double disposition dans les plis sur plusieurs Unio de l'Amérique du Nord; nous avons pu le vérifier sur des exemplaires des Unio undulatus el tuberculatus. Barnes et de l'U. naviformis. Lamk. qui vivent dans l'Ohio. 
Coquille ovale, épaisse, ventrue, relevée à sa partie postéricure de plis de deux ordres commic dans la précédente; les plus marqués partent de derrière les sommets pour se porter jusques aux bords inférieur et postéricur. Les sommets sont proéminents, un peu recourbés vers le corcelet et placés au tiers antérieur de la coquille. Le bord inférieur est sinué, comme crénelé postérieurement par l'effet de la terminaison des plis principaux; le bord supérieur est arqué. Les stries d'accroissement sont rapprochées.

On distingue celte coquille de la précédente par sa taille moindre, par son épaisseur plus forte, par sa forme générale, courte, bombée, enfin par les grands plis dont quelques-uns se purtent plus en avant et rendent le bord inférieur crénelé dans sa partie postérieure.

Localités : Laymont, le Pin, le Planté, Lahas, Nizas, etc.

3. Mulette subtrigone. Unio sublrigonus. Noul.

Pl. 3, fig. 2, 3.

U. Testâ subtrigonâ, crassissimâ, plicatâ; plicis latis ì natibus ad partem totam posteriorem radiantibus, natibus utmidis, prominentibus ; margine superiore arcuato, inferiore sublineari; dente cardinali crasso, triangulari-compresso.

Alt. $0^{\mathrm{m}}, 04.5$.

Lat. 0,075 .

Coquille très-épaisse, subtrigone, à sommets élevés, d'où partent, en se déployant en éventail, des plis qui occupent en totalité la partie postérieure de la coquille; ces plis sont représentés par des gouttières profondes à l'intérieur. Les stries d'accroissement sont très-prononcées. La dent cardinale est triangulaire, un peu comprimée.

Localités : Laymont, le Planté, Lahas.

4. Mulette a larges plis. Unio latiplicalus. Noul.

U. Testâ ovato-elongatâ, crassâ, ventricosâ, plicatá; plicis 3, 4, latis, summo in uno confertis à natibus ad margincm posteriorem decurrentibus, plicis alleris, vix 
anticè prominulis, versìs marginem superiorem decurrentibus; natibus latis, lumidis, prominentibus; margine inferiore subsinuato, margine superiore arcuato; dente cardinali crasso....

$$
\begin{aligned}
& \text { Alt. } 0^{\mathrm{m}}, 05 . \\
& \text { Lat. } 0,1 .
\end{aligned}
$$

Coquille épaisse, ventrue au milieu, surtout immédiatement au-dessus de la naissance des grands plis. Ceux-ci, plus larges à leur terminaison que ceux des autres espèces, se portent tous jusqu'à la marge de la partie postérieure; les plis qui se dirigent vers la charnière ne se montrent qu'en avant et sont à peine indiqués. Les stries d'accroissement sont trèsespacées et fortement prononcées.

Je n'ai pu rétablir entièrement cette coquille, surtout à l'intérieur; néanmoins, un fragment de moule m'a permis de constater la forme générale de la dent cardinale qui était épaisse et tuberculeuse.

Localités : Pessan, dans une sablière, avec des os de Rhinoceros et de Mastodonte, (M. Lartet.) à Pellefigue, près de Simorre, (M. Dupuy.) à Lahas, à Nizas, etc.

5. Mulette a Plis ètrorts. Unio strictiplicatus. Noul.

$$
\text { Pl. } 1 \text {, fig. } 2 .
$$

U. Testâ ovatâ, crassâ, plicatâ; plicis strictis, 3 libcris à margine inferiore et posteriore versìs nates decurrentibus, plicis alteris numerosis, recurvis, ad marginem superiorem et posteriorem decurrentibus; natibus latis, tumidis, prominentibus; margine inferiore sinuato, superiore arcuato; dente cardinali crasso, conico-triangulari, obtuso, sulcato.

$$
\begin{aligned}
& \text { Alt. } 0^{\mathrm{m}}, 05 \text {. } \\
& \text { Lat. } 0,08 \text {. }
\end{aligned}
$$

Cette coquille varie peu par sa forme générale; on la distingue facilement à ses sommets très-étendus, et par suite de cette disposition à l'écartement des principaux plis, qui, au lieu d'avoir une origine commune, naissent séparément. Les 
plis sont très-étroits et séparés par de larges gouttières, ce qui est le contraire dans les autres espèces plissées. Le bord supéricur est fortement arqué. Je ne connais que la dent cardinale du côté gauche, quì m'a paru à peu près semblable à celle de l'Unio breviplicatus.

Localités : Laymont, le Planté, le Pin.

Ors. Il faut peut-être rapporter à cette espèce une coquille qui m'est connuc par un moule extéricur presque complet et qui présente aussi des gouttières larges séparées par des côtes étroites. Mais dans celle-ci, les gouttières et les trois côtes principales sont recourbées en avant et dirigées vers le bord inféricur de la valve, au licu d'être relevées vers le haut. Ce moule vient de Laymont.

\section{B. Non plicati.}

6. Mulette de Lacaze. Unio Lacazi. Noul.

Unio pictorum, Var. Lacaziana. Dupux, Moll. du Ger's, p. 102. (Non Unio piclurum. Auct.)

$$
\text { Pl. 4, fig. 1, } 2,3,4 \text {. }
$$

U. Testâ ovato-oblong $\hat{a}$, tenui, anteriùs rotundatâ, posteriùs subangulatâ; natibus subprominentibus; margine superiore lineari, vix arcuato, margine inferiore lineari aut subsinuato; dente cardinali conico-tuberculato.

Alt. $0^{\mathrm{m}}, 03$.

Lat. 0, 06 .

B. Atténuée. Attenuatus. Noul.

Testâ longiore, posticè attenuatâ.

Pl. 5. fig. 1, 2.

Alt. $0^{\mathrm{m}}, 03$.

Lat. $0,07$.

La coquille de cette espèce est ovale allongée, arrondie à son extrémité antérieure, un peu anguleuse postérieurement. Le bord supérieur est très-peu arqué, presque droit; l'inférieur est 
DE L'ACADÉMIE DES SCIENCES.

droit ou à peine sinué. Les sommets rapprochés de l'extrémité antérieure, sont arrondis et un peu proéminents. Les stries d'accroissement sont assez prononcées. La dent cardinale est tuberculeuse et de forme conoïde.

La variété est plus longue et présente l'extrémité postérieure sensiblement rétrécie.

Le caractère de la dent cardinale de celte coquille la sépare suffisamment de l'Unio pictorum. Drap. dont la dent est aplatie en crête, et aussi de l'Unio Requienii. Mrcr. , qui est l'espèce qui vit si commune dans toutes nos rivières $d a$ bassin souspyrénéen (1) et qui offre la dent cardinale parfois légèrement tuberculće, mais furt éloignée de celle de l'Unio Lacazi.

Localités : Cologne (Gers), Saint-George, où M. Lacaze, à qui je dédie cette espèce, la recucillit le premier; Laymont, le Pin, etc.

7. Mulette de Laymont. Unio Laymontianus. Noul.

Pl. 6, fig. 1, 2.

U. Testâ ovato-oblongâ, subreniformi, crassâ, anteriùs rotundatâ, posteriùs vix attenuatâ; natibus prominentibus; margine superiore arcuato, inferiore sinuato; dente cardinali tuberculato, irregulariter conico, summo sulcato.

Alt. $0 \mathrm{~m}, 04$.
Lat. $0,083$.

Coquille ovale-oblongue, supérieurement arquée, à bord inférieur sinué. Les sommets sont saillants; à l’intéricur les impressions palleales, éloignées de la marge, sont très-prononcées. La dent cardinale est grosse, tuberculeuse, conoïde, striée comme déchirée au sommet.

Localités: Laymont, à l'état de géode; Cologne, où les empreintes sont dans un grès à petits grains très-durs.

(1) L'espèce vivante qui se trouve décrile dans mon précis de l'Hist. des Moll. du Bassin sous-pyr., pag. 77, sous le nom d'Unio pictorum. Disp., et qui a été énumérée sous cette même dénomination par M. Dupuy, dans son Essai sur les Moll. du Gers, pag. 78, doit être rapportée à l'Unio Requienii. MI I 
8. Mulette de Lartet. Unio Larteti. Noul. PI. ๖, fig. 3.

U. Testî̀ ovalâ, compressâ, tenui, anteriùs rotundatâ, posteriùs subangulata; margine superiore sublineari, inferiore arcuato; natibus prominentibus; dente cardinali?

$$
\begin{aligned}
& \text { Alt. } 0 \mathrm{~m}, 02 . \\
& \text { Lat. } 0,035 .
\end{aligned}
$$

Cette coquille, dont nous devons la découverte et la communication à M. Ed. Lartet, est la plus petite de nos Mulettes. Elle a des rapports avec les jeunes individus de l'Unio littoralis, subtetragona de nos rivières. Nous ne connaissons encore que son extérieur, l'ayant toujours vue empâtée dans le calcaire durci qui lui sert de gangue. Le test est ordinairement conservé; il est mince et offre les stries d'accroissement fines, sinueuses et très-rapprochées.

Localités : Seissan, avec la Mélanic d'Aquitaine, dans un filon de calcaire, aujourd'hui épuisé, d’après M. Lartet.

9. Mulette Anodontö̈de. Unio Anodontö̈des. Nuul.

Très-grande coquille que nous ne pouvons caractériser complétement, ne possédant que des fragments de plusieurs moules insuffisants à la rétablir d'une manière précise, quoiqu'ils laissent deviner sa forme générale. Elle devait avoir à peu près les dimensions de l'Anodonta cygnea. Drap.' Les stries d'accroissement étaient fortement écartées et saillantes.

Localités : Lahas, Pessan, avec quelques-unes des espèces plissées.

Sans vouloir, celte fois, entrer dans la discussion théorique des faits consignés dans le présent mémoire, je me contenterai d'en tirer les conclusions suivantes :

1. Les couches sableuses, plus rarement les couches argileuses du terrain miocène, dans la partie aquitanique du bassin sous-pyrénéen, renferment une population de coquilles d'espèces perdues appartenant aux genres Mélanie et Mulette. 
DE L'ACADÉML DES SCIE.TCES.

Un grand nombre d'espèces de ces deux groupes vivent actuellement dans les eaux douces d'Europe, et souvent ensemble dans les rivières et les grands lacs de l'Amérique du Nord. Jusqu'ici on n'a trouvé que dans cette partie du NouveauMonde des Mulettes relevées à leur surface de plis ou de côtes saillantes.

$2 .^{\circ}$ Les mêmes couches du terrain miocène sous-pyrénéen qui recèlent nos coquilles, contiennent des ossemens fossiles de nombreux animaux vertébrés; nous sommes donc autorisés à regarder tous ces débris comme contemporains, et à considérer leur dispersion dans ces couches sédimentaires comme due aux mêmes causes.

3. ${ }^{\circ}$ Les valves des Mulettes qui ont laissé des empreintes se montrent le plus souvent isolées, quelquefois fragmentées, très-rarement réunies par paires. Leur séparation el leur état de conservation témoignent de la mort des individus auxquels elles avaient appartenu et du transport plus ou moins éloigné de ces parties solides, au moment de leur délaissement.

$4 .{ }^{\circ}$ L'existence de nos mollusques fut certainement de longue durée, puisque leurs débris solides ont laissé des traces à différentes profondeurs dans l'épaisseur des collines qui séparent les vallées que parcourent les petites rivières du Gers.

Je dois ajouter que les couches du terrain miocène, soit argilcuses, soit sableuses, recèlent plusieurs espèces du genre Hélice (Helix), mais que je n'ai encore rencontrées qu'à l'état de moules intérieurs, le plus souvent incomplets, incapables par conséquent de fournir des caractères suffisants pour les faire distinguer convenablement comme espèces. 


\section{FXPLICATION DES FIGURES,}

QUI TOUTES SONT DE GRANDEUR NATURELLE.

\section{PLANCHE 1.}

Mélanie d’Aquitaine. Melania aquitanica.

1. La coquille vue dans sa position naturelle el présentant l'ouverture.

2. La même du côté opposé.

\section{PLANCHE 2.}

Mulette en Éventail. Unio flabelliferus.

1. Valve gauche vue à l'extérieur.

Mulette a plis Étrorts. Unio strictiplicatus.

2. Valve droite vue à l'extérieur.

\section{PLANCHE 5.}

Mulette a plis courts. Unio breviplicatus.

1. Valve gauche vue à l'extérieur.

Mulette subtrigone. Unio subtrigonus.

2. Valve gauche vue à l'extérieur.

3. Valve droite vue à l'intérieur.

PLA NCHE 4.

Mulette de Lacaze. Unio Lacazi.

1-4. Valves dépareillées, dont deux vues à l'extérieur et deux à l'intérieur.

\section{PLANChE s.}

Múlette de Lacaze, atténuée.

1-2. Valves dépareillées, l'une vue à l'extérieur, l'autre à l'intérieur.

Mulette de Laktet. Unio Larteti.

3. Valve droite vue à l'extérieur.

PLANCHE 6.

Mulette de Laymont. Unio Laymontiana.

' Valves dépareillées vues à l'intérieur.

1. Valve gauche.

2. Valve droite. 


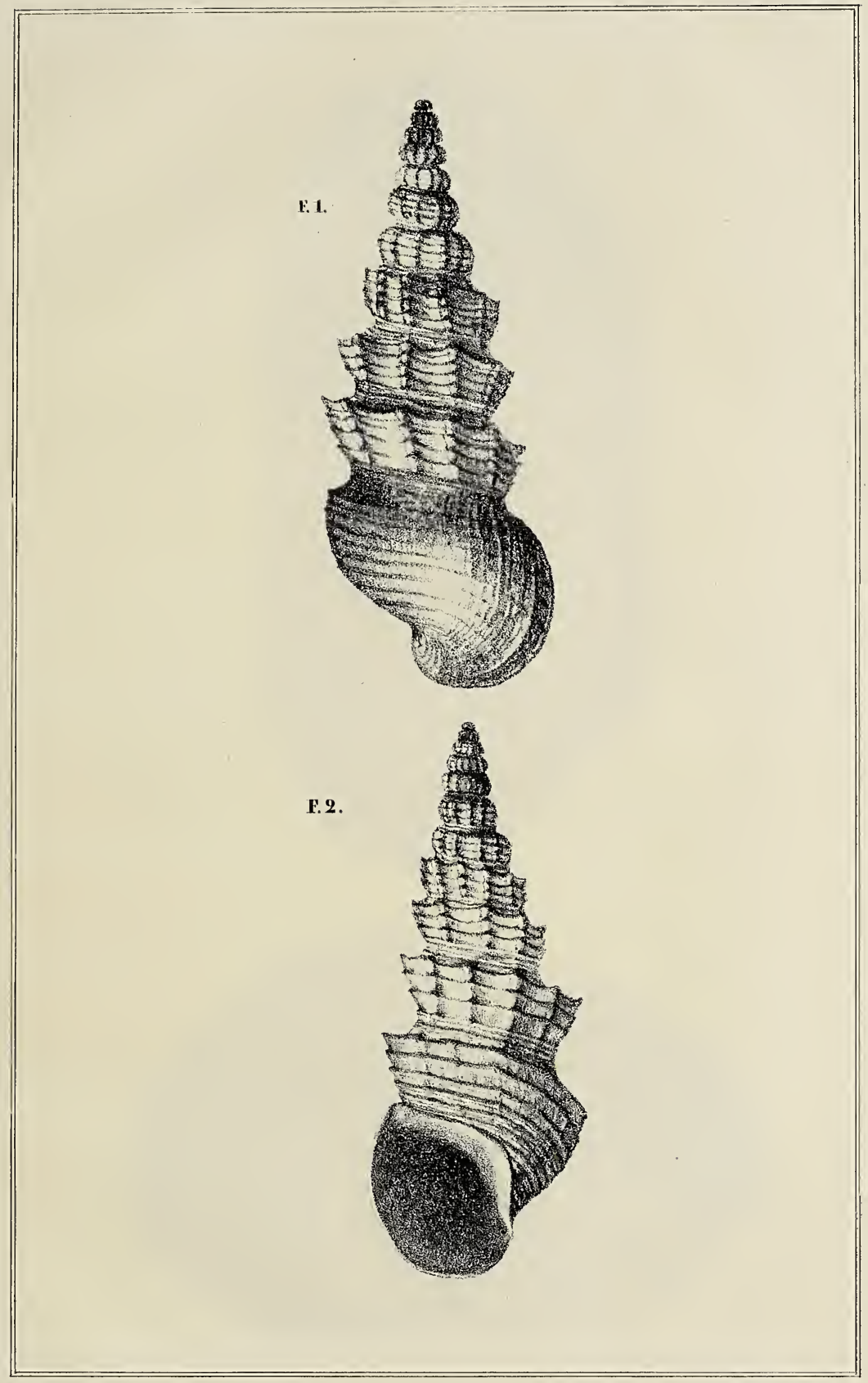

L.Lacaze, del.

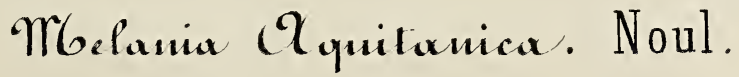





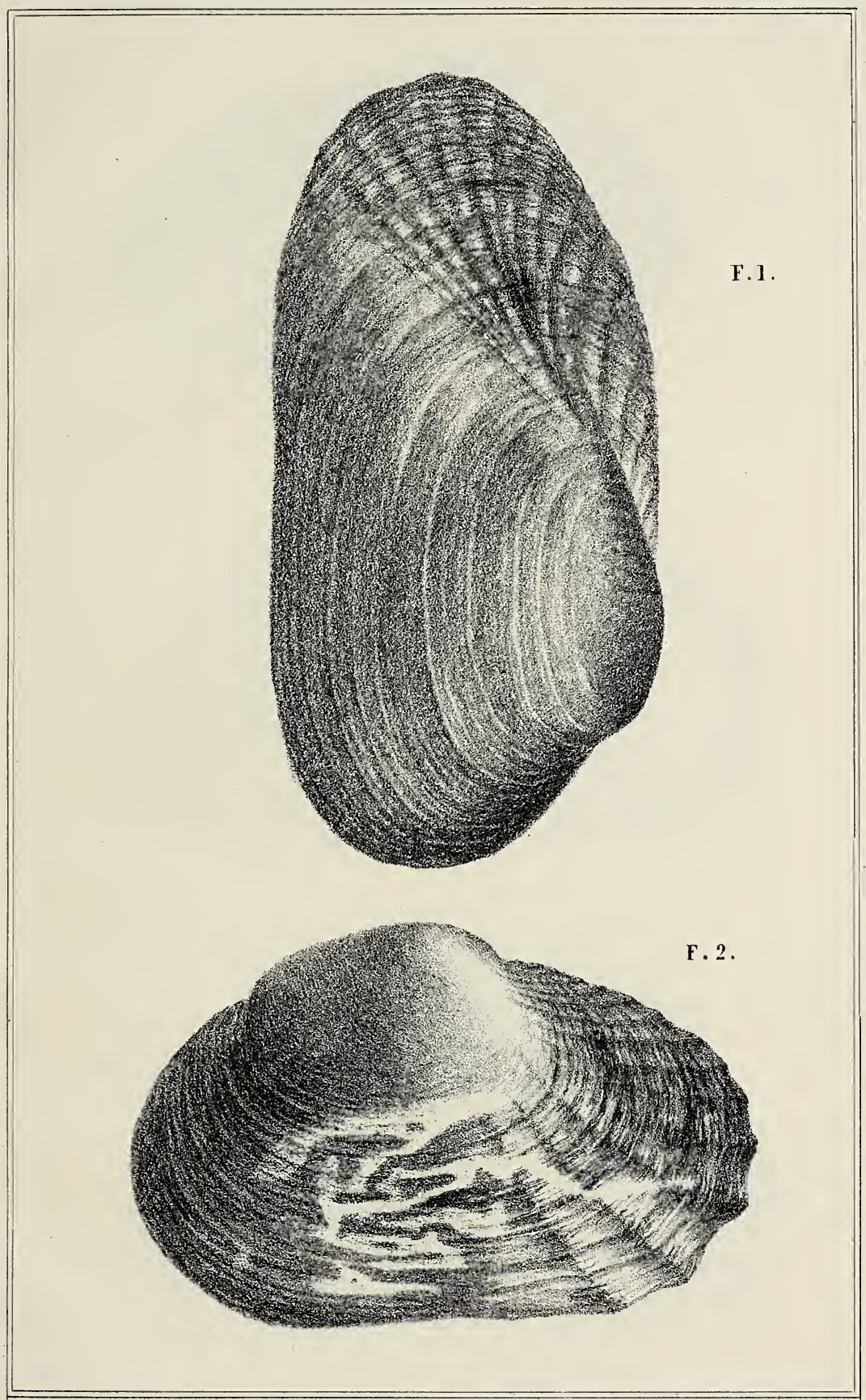

Lith. Delor à Touivuse
1. Il niv Trabepliferus. Noul.
2. Iluio Strictiplicartus. Noul. 



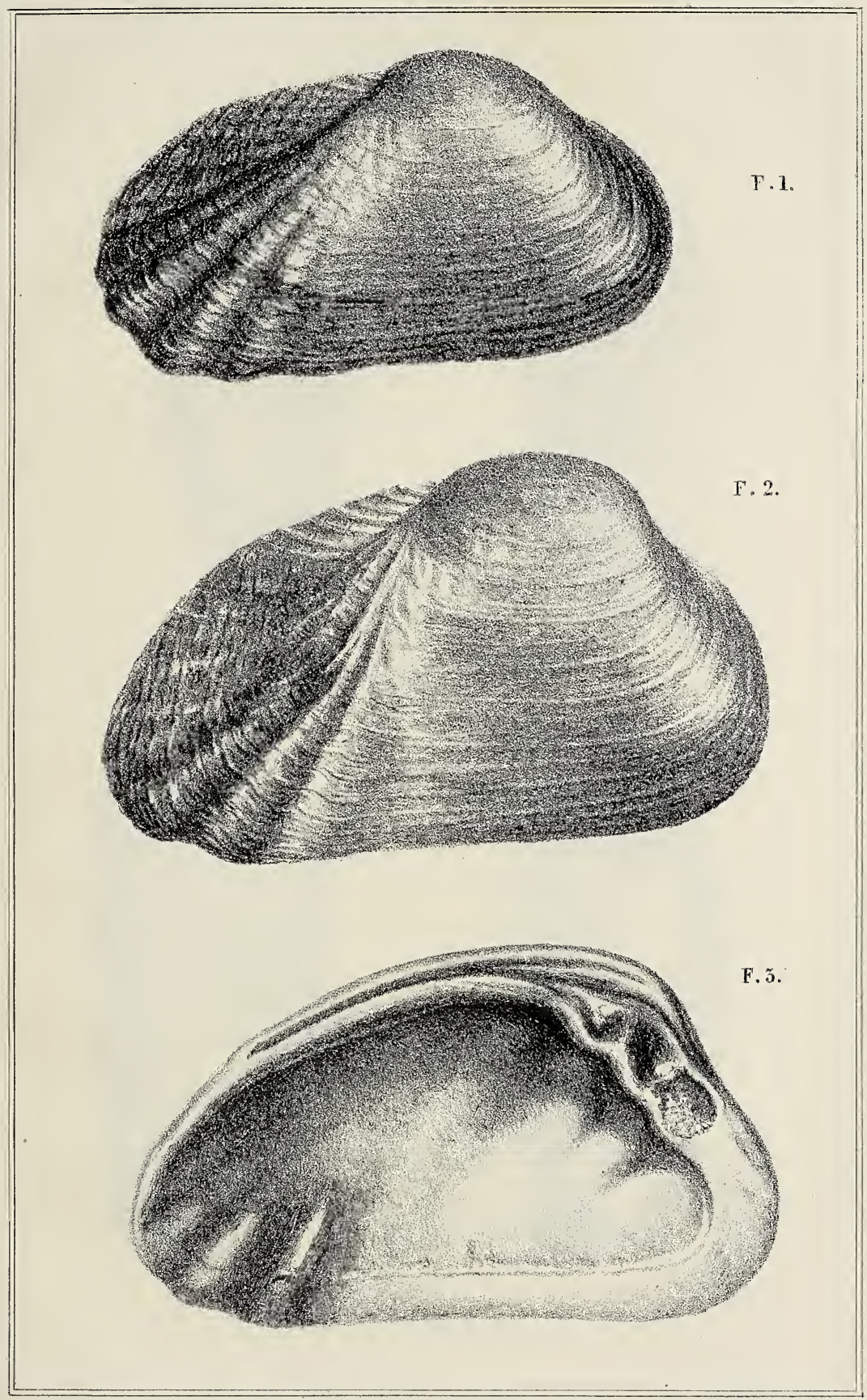

L. Lacaze, del. Iith. Delor a Ioulouse
1. "lluis SBaviplicartus. Noul.
?-3. Uniw Subtrigonus. Noul. 



$$
\begin{aligned}
& 01 \\
& 00
\end{aligned}
$$



F. 2

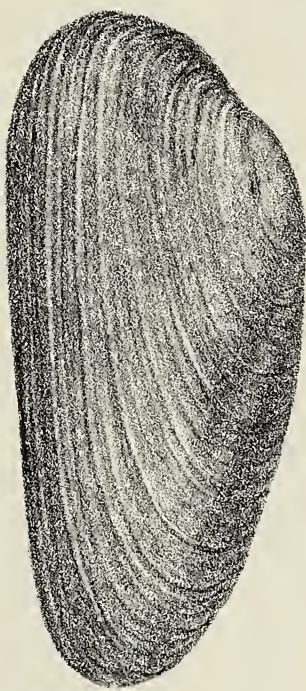

F. 1

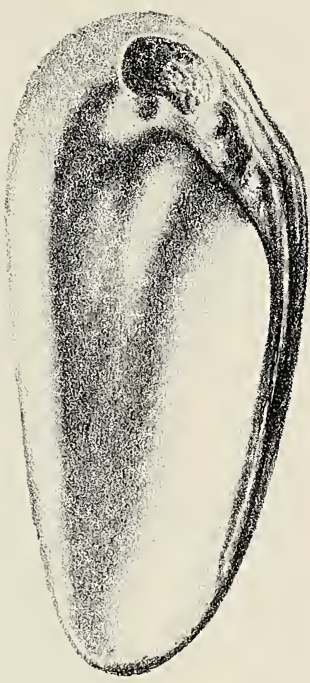

F. 3.

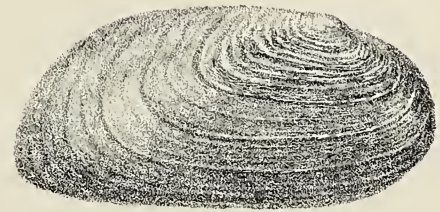

L. Lacaze de1.

Lith.Delor à Toulouse.

1-9. Iluio Racasi, Var. Noul.

3. Lluiv facteli. Noul. 

P1. G.

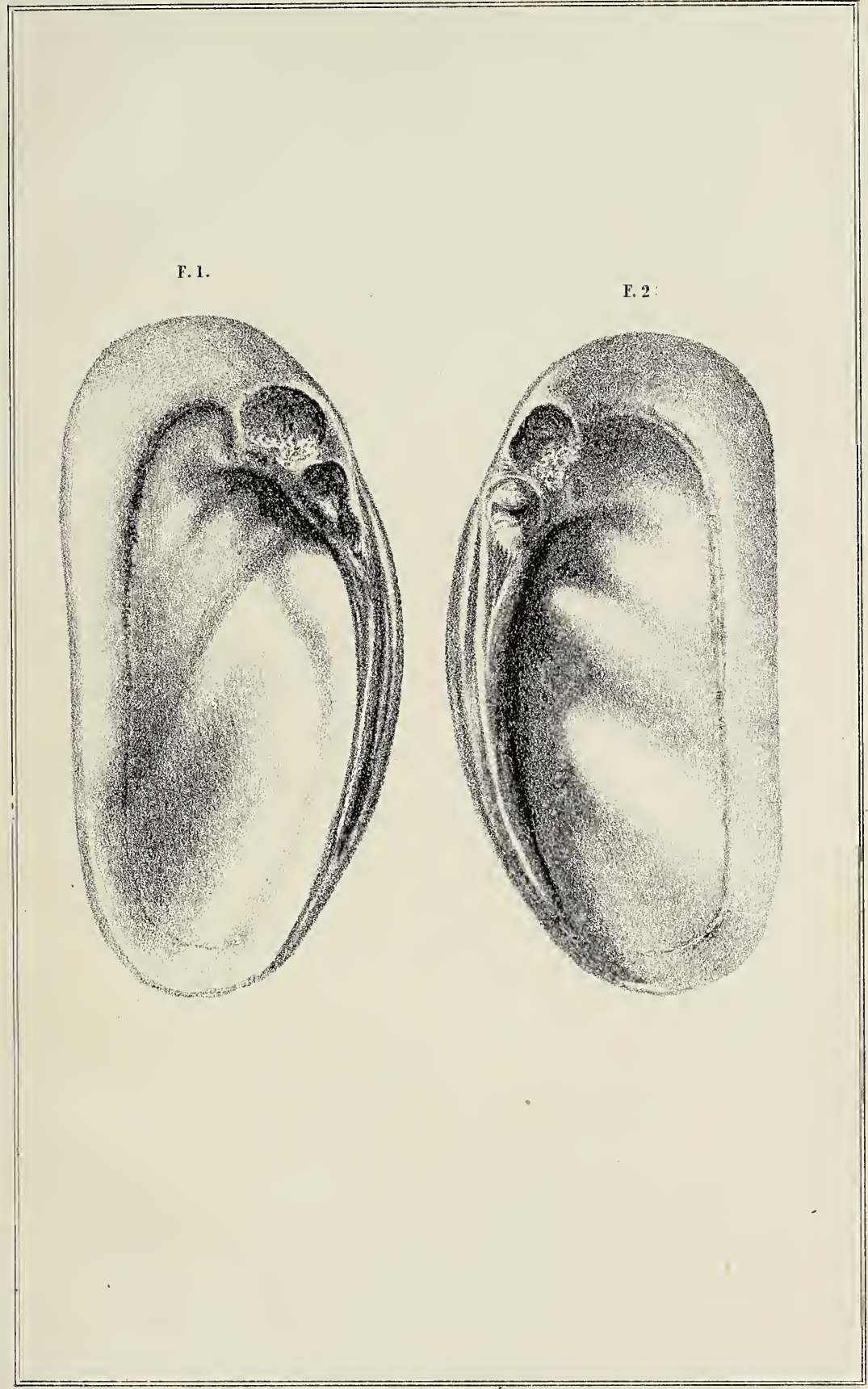

L. Iacaze, del.

Lith. Delor a Toulouse 1-2. Il niv Paymontianus. Noul. 






Go. Coshy'gi

With illo of posid sheth m 6 hitho p. plates by

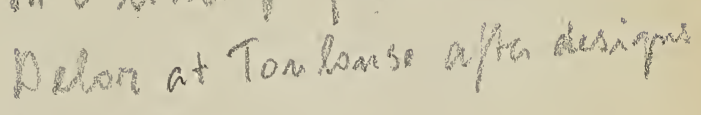
by L. Lacaze.

Pp. 225.240.

Uxthat from the nemones

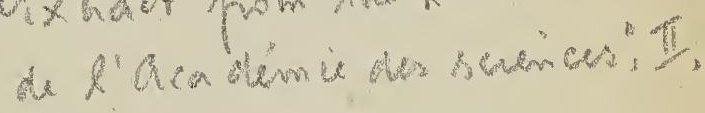
1844. 
5335

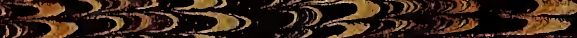

$32.35,5355$

D. $\frac{15}{3}$

3) 23535

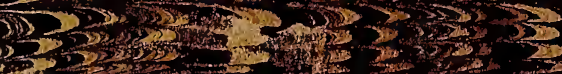

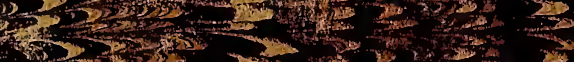

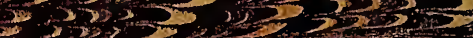

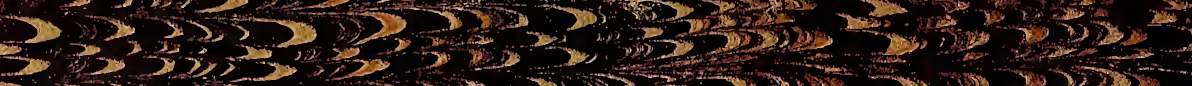

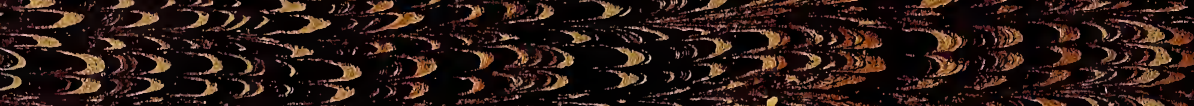

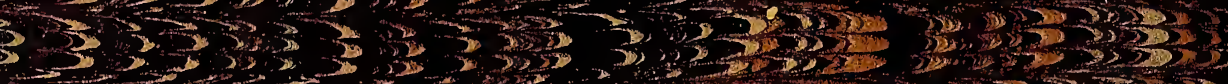

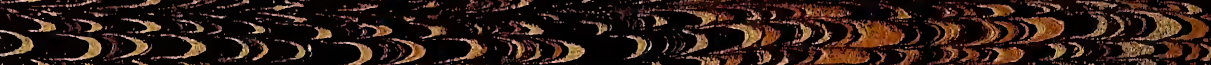

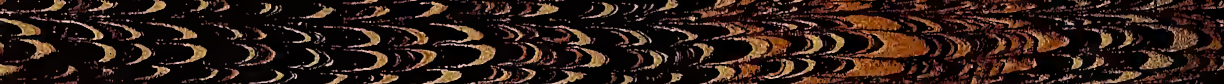

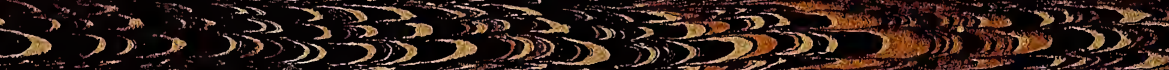

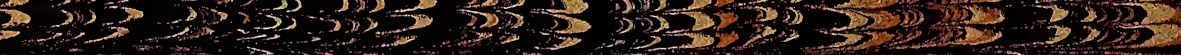

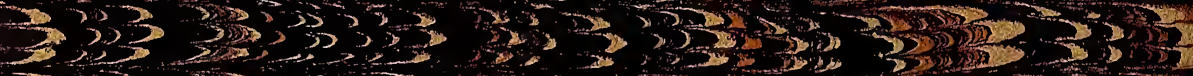

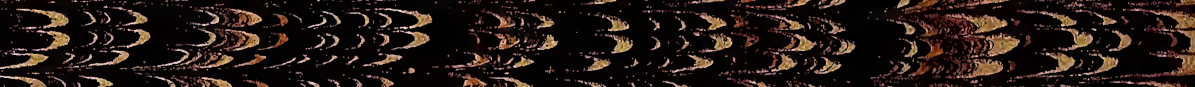

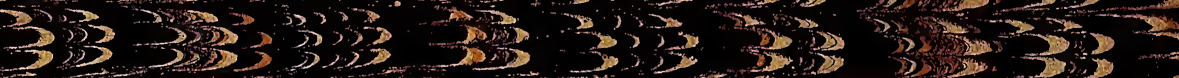

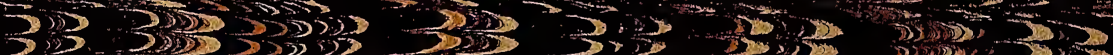

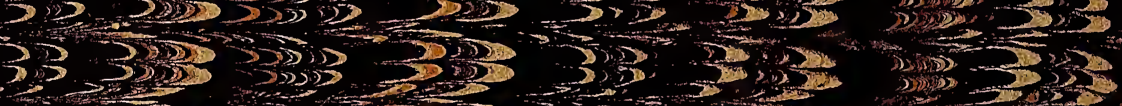

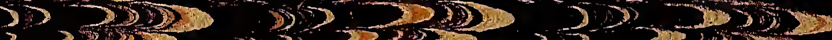
$33,3,35$ (i) $\rightarrow$ S $\rightarrow 20$ $\rightarrow+23$ $\sin _{3} 25$ $D_{3} \rightarrow 353$ $3,20353,3, \frac{23}{2}, 3,5$ $3.353,3,3,3,353$ in 273 3z. 3,33,

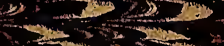

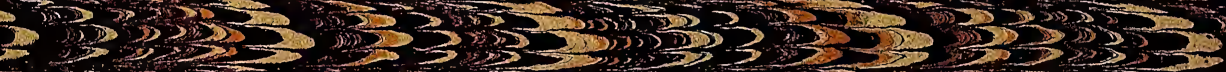
5325

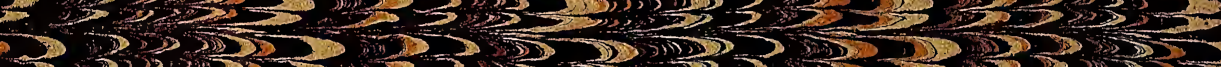

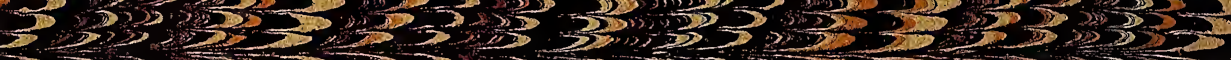

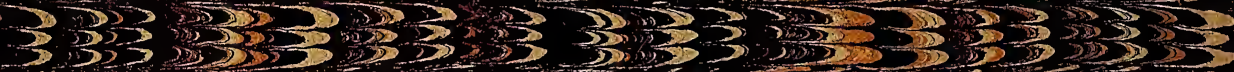

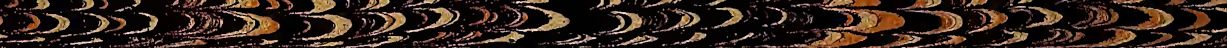

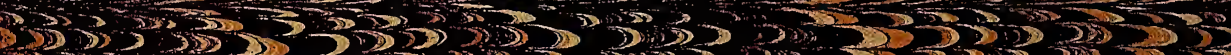

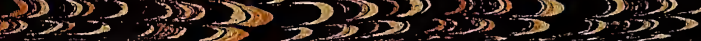

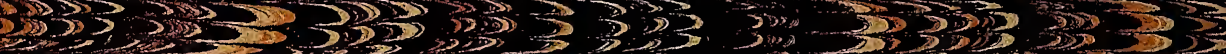

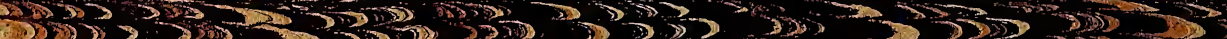

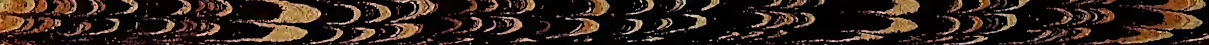

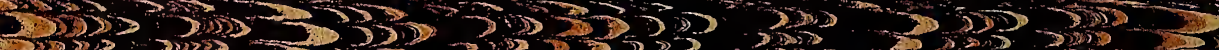

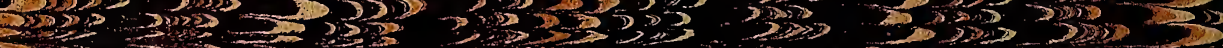

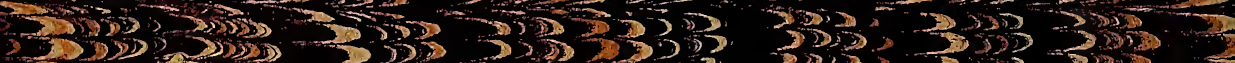

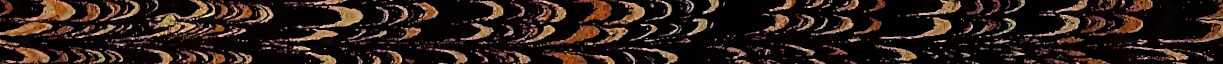

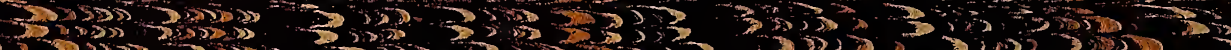

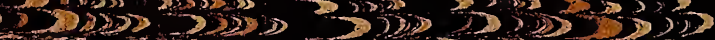

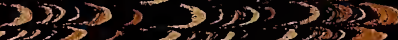

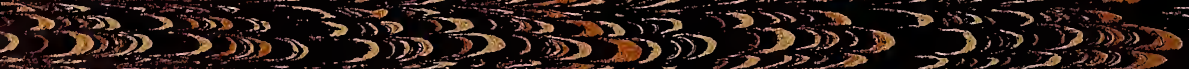




\section{$x^{2}$}

it
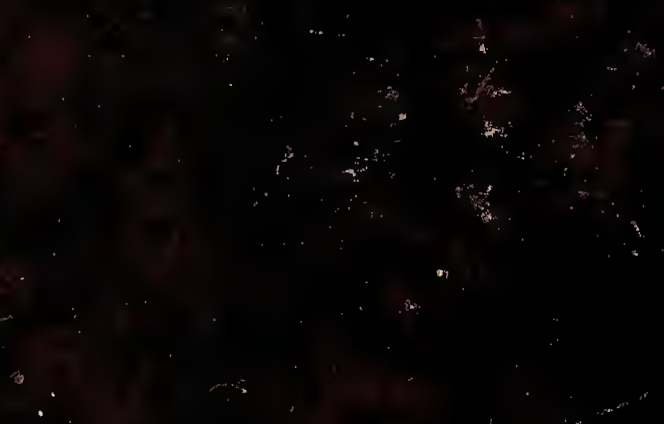

to

$$
x+2
$$

$-1,3$

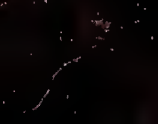

3.
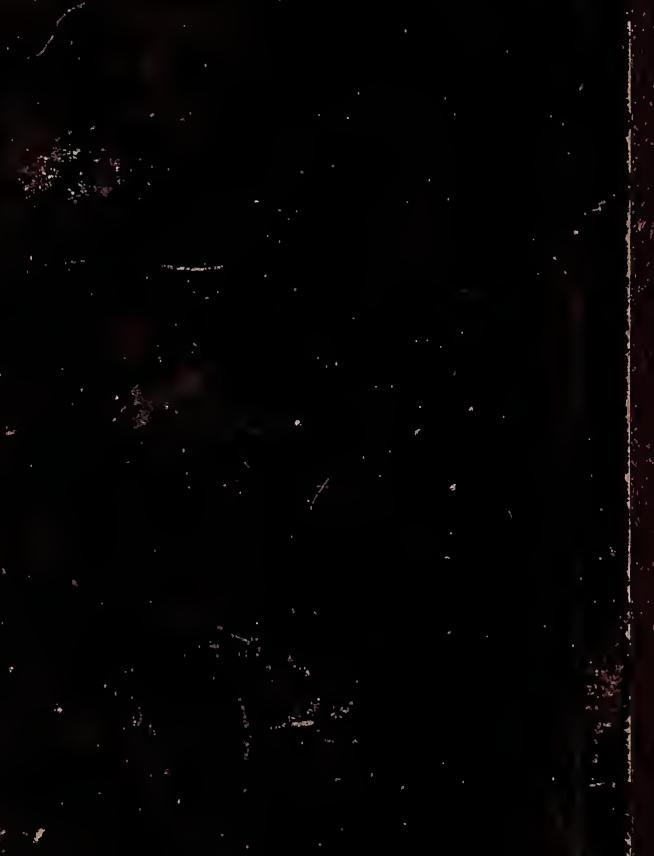

$\lambda$ 